\title{
86. Photoperiodic Initiation of Flower Primordia in Japanese Morning Glory, Pharbitis Nil Chois.
}

\author{
By Shun-ichiro IMAMURA \\ Laboratory of Applied Botany, Faculty of Agriculture, Kyoto University \\ (Comm. by H. Kihara, M.J.A., July 13, 1953)
}

The studies of photoperiodic responses in Japanese morning glory, undertaken some time ago under the guidance of Dr. Kwan Koriba in the Botanical Institute of Kyoto University, came for the time being to a conclusion. In the present brief article the main results are summarily reported.

1) The Japanese morning glory, Pharbitis Nil Chois. is known as one of the most sensitive short day plants ${ }^{1225 j 68}$. The strain used in the present study has been obtained from Dr. H. Kihara. The plants remain under continuous illumination strictly vegetative for months, and may attain a large size without differentiating a single flower primordium. When transferred to short day conditions, the strain responds very readily to photoperiodic stimulus by initiating flower buds. Even seedlings, having only cotyledons, which did not yet fully expand the foliage leaves are capable of responding to photoperiods.

Under favorable conditions even a single relatively long dark period will induce the plants to initiate flower primordia. No more consecutive dark periods are necessary, and the buds in the axils of the developing leaves differentiate into flower primordia. If the stimulus given is not strong enough, the induction is reversible. After the growing point has given rise to several axillary flower primordia, it becomes again vegetative and continues to develop foliage leaves with vegetative axillary buds. In such a case the number of flower primordia initiated can be used as a measure of photoperiodic response. When long dark periods are given repeatedly, the terminal bud begins to form bracteal leaves and, since its growing point is used up to form a terminal flower primordium, the apical growth of the stem stops. The number of plants with terminal flower buds can also be used as another measure for the intensity of photoperiodic induction.

2) When a topped plant is deprived from all leaves except for the just expanded one, the axillary bud of this leaf begins to grow into a lateral shoot. If such a plant is subjected to long dark periods, the developing axillary shoot differentiates lateral flower buds only or, in addition, also a terminal flower bud, according to the intensity of the stimulus given. In favorable conditions a topped 
plant with a single leaf can be induced to initiate flower primordia even by a single dark treatment.

During the investigation reported here many experiments were carried out with topped plants, with the result that the response manifested itself in the axillary buds of the developing lateral shoot. In the following, the leaf to which the stimulus is given will be called "donor leaf". The "receptor buds", i.e. the buds which respond to the stimulus, can be also restricted in number, as one wishes, by removing the superfluous buds. In most cases the axillary bud of the donor leaf was used as a receptor bud, but one or more other buds were also left intact and used as receptors. By this procedure the photoperiodic response can be analysed more precisely, because one can control at will the locus of perception of the stimulus and the site of morphogenous response.

3) When the uppermost leaf of a topped plant is used as donor leaf and its axillary bud as receptor, the latter begins to grow immediately after the removal of the top. If the donor leaves are kept in darkness of adequate duration, the receptor buds are induced to initiate flower primordia. The position of the initiated flower buds on the developing receptor shoot shows some variation, according to the age of the bud itself, to the position or age of the donor leaf and, moreover, to the time of starting with dark period treatments after topping. The later the treatment is started, the higher is the node situated, at which the flower primordia appear. This fact shows that some of the lower lateral buds of the receptor shoot had been already determined as vegetative buds, before the stimulus reached to the receptor bud, and could not be changed by subsequent dark period treatment.

4) Flower primordia can be initiated by subjecting the whole plant or a single donor leaf to a dark period of longer duration than the critical dark period, i.e. about 8-9 hours. With a longer dark period than the critical, the increasing duration of treatment increases the number of plants responding to it and, also, the number of the flower primordia initiated. A shorter dark treatment than the critical cannot induce the plants to initiate any flower primordia, even if the treatment is given repeatedly. By a continuous darkening of the donor leaf, initiation of flower primordia can be induced.

Sensitivity of the plants to dark period treatment varies markedly according to the external and internal conditions. In midsummer when the temperature in the green house is very high, plants show a low sensitivity, whereas in early and late summer they respond to the darkness more readily. The age of the donor leaf is also of great importance. In young plants with 3 mature 
leaves, the third leaf is the most sensitive, while older leaves have a low sensitivity. The expanding leaves show varying sensitivity in their various developmental stages. In very young, developing leaves no induction occurs.

5) Completely defoliated plants cannot be induced to initiate flower primordia by subjecting them to darkness. Illumination of the shoot axis, receptor bud and petiole of the donor leaf have no effect upon the reaction. Only the leaf blade is the site of perception of the stimulus. A reduction of the area of the donor leaf, by cutting off the apical and lateral parts of the blade, decreases the induction. With increasing leaf area the number of the flower primordia initiated increases.

If a portion of the donor leaf is subjected to darkness by being enclosed in a bag of light-tight paper and its remaining parts are exposed to light, flower primordia can be initiated. But the number of primordia is less than when the whole donor leaf is darkened. Dark period treatment of the basal half of the leaf seems to be more effective in the initiation of flowers than that of the apical half.

6) The exposure of plants to light of very short duration in the middle of a long dark period, the so-called "light break", can diminish or nullify the effect of the dark treatment. When two successive dark periods of subcritical duration for example 7 hours -are applied successively to the apical and then immediately hereafter to the basal half of the same donor leaf, and this is repeated for several days, no flower primordia or a few only are initiated. The effect is very weak in contrast to plants whose basal or apical half of the donor leaf is subjected daily to one dark period of double duration - for example 14 hours. Two successive inductions caused by dark periods of subcritical duration applied to two different parts of one leaf can thus hardly add up to the induction of critical length.

7) If the donor leaf is removed immediately after a dark treatment of longer duration than the critical, for example from 10 to 16. hours, no flower initiation occurs. But when the duration of the dark treatment exceeds 18 hours or more, the removal of the donor leaf cannot suppress the appearance of flower primordia on the receptor bud. The term "critical transmission period" is used in the following to designate the time elapsing from the commencement of the dark treatment until the removal of the donor leaf, during which an induction seems to be transmitted from the donor to the receptor with sufficient intensity to cause initiation of flower primordia. The critical transmission period is dependent chiefly 
upon the distance and mutual position of the donor leaf and the receptor bud. In experiments with a young just fully expanded leaf as donor and its axillary bud as receptor, this period is about 18 hours in most cases.

8) Not only induction is transmitted from one branch to another of the same plant but also from one induced plant to another non-induced one which is grafted to the former and maintained on non-inductive light conditions. The transmission from the donor to the receptor occurs more readily when the donor is deprived of its developing buds and the receptor of its leaves.

9) The path of the transmission of induction seems to lead through living tissue. Scalding by means of a hot wire, heated by electric current, applied in the path leading from the donor leaf to the receptor bud shows that the induction cannot traverse the dead tissue. Incisions into the stem do not inhibit floral initiation but retard transmission of induction, as revealed by the initiation of flower primordia at higher nodes than in the control plants.

10) The velocity with which the induction is transmitted along the plant seems to be rather small. It was measured in the following manner. Dark treatment was given to two branched plants having a donor leaf on one branch and a receptor bud on another one. Besides the experimental lot, several other lots of plants with a donor leaf and its axillary bud as receptor were used as timescale. They received the same dark period treatment as the experimental lot but at various intervals, namely, some of them immediately and others one, two, three or more days after the removal of the top. The higher are the flower initiating nodes located on the stem of the receptor, the later does the stimulus reach to the bud. (See 3.) As the distance between the donor leaf and the receptor bud in the experimental plants was larger than that in the timescale plants, the primordia of the former appeared at higher nodes than those of the time-scale lot, which have received dark treatment immediately after topping, and their position falls between that of two of the time-scale lots. The time required for the transmission of the induction to the indicator bud in enough intensity to cause floral initiation can be calculated by means of interpolation. This procedure cannot claim high accuracy but may be used as a method of first approximation. In this way not only the transmission velocity from one branch to another in two branched plants was determined but also that from a donor plant to a receptor plant grafted to it. The induction moves along the stem with the average velocity of $2.0-3.8 \mathrm{~mm}$ per hour

11) Polarity in the transmission of the stimulus was also 
studied by the method described in the preceding paragraph. The stimulus moves acropetally more quickly than in the opposite direction. The number of flower primordia on the receptor is variable, according to whether the receptor bud is situated on a higher or on a lower node than the donor leaf. The lower buds, in contrast to the higher, develop a smaller number of flower primordia. Often no flowers are initiated on the lower receptor bud.

12) As in many other short day plants illumination of the donor leaf in the middle of the dark period diminishes or nullifies the effect of dark treatment. The intensity of light preceding the dark treatment is also of great importance. Plants exposed to light of very low intensity, i.e. 0.1-0.2 foot candle, for 24 hours before the dark treatment, can hardly react to the latter. This fact indicates that not only the changes or conditions brought about by darkness but also the changes or conditions attributable to light, are involved in photoperiodic initiation of flower primordia ${ }^{33}$. The "light period reaction" and the "dark period reaction" as one may designate them, must take place successively in the same leaf or in the same part of a leaf, in order to cause floral initiation. By exposing the plants to a light of very low intensity the light period reaction is disturbed; such plants can not be induced to floral initiation by subsequent dark treatment, unless before dark treatment they are exposed to light of sufficient intensity for several hours. When the basal half of the donor leaf in which the light period reaction has been disturbed by exposing it to light of low intensity is covered and the apical half exposed to natural day light, no flower initiation takes place. The light period reaction taking place in the exposed apical half of the donor leaf and the dark period reaction, which may occur in the basal half, cannot therefore add up to bring about floral initiation.

13) Upon the removal of the donor leaf at the time when the critical transmission period is just over, only a few flower primordia are initiated, but if the donor leaf is removed several hours thereafter, the flower primordia increase considerably in number. The condition of the donor leaf, whether, it is kept in darkness or illuminated, after the critical transmission period until defoliation, has no serious effect upon the number of primordia initiated. The experiment indicates that the leaf, once induced, can continuously supply a florigenic stimulus to the receptor bud even in the light.

14) Plants to which several short day photoperiods are given are so stable in their state of induction that they can hardly be reverted to the strictly vegetative condition by being maintained in continuous light. Even if all the leaves induced and all develop- 
ing axillary buds are completely removed, the growing point of the subsidiary bud which appears later, maintains the induction to initiate flower primordia for a long time. The after-effect of repeated short day treatments lasts, at least, several weeks or months.

15) The results obtained show that the photoperiodic response in Pharbitis Nil is mainly the same as in Xanthium Pennsylvanicum ${ }^{4}$, and it seems highly probable that some substance or substances are involved in the processes of floral initiation. The light conditions may influence floral induction through their action on the synthesis and distribution of the involved substance. It was proved that the substance in question must be distinct from auxin, as it moves in the stem not only in basipetal but also, even more quickly, in acropetal direction. The velocity of transmission is much smaller than that of auxin reported in many plants.

The author wishes to express his sincere thanks to Dr. K. Koriba for his kind guidance and criticisms.

\section{References}

1) Asanuma, K.: Influence of day length on growth and flowering in Pharbitis Nil Chois. Studies from the Institute of Horticulture, Kyoto Imperial University, No. 1, 206-233 (1934) (in Jap.).

2) Eguchi, T.: Effects of the day-length upon the time of differentiation of flower bud and the subsequent development to flowering. Proc. Imp. Acad., 13, 332333 (1937).

3) Hamner, K. C.: Interaction of light and darkness in photoperiodic induction. Bot. Gaz., 101, 658-687 (1940).

4) Hamner, K.C. and Bonner, J.: Photoperiodism in relation to hormones as factors in floral initiation and development. Bot. Gaz., 10C, 388-431 (1938).

5) Nakayama, S. : Studies on flower initiation. II. Seitaigaku-kenkyu, 12, 4851 (1948) (in Jap.).

6) Yoshii, Y. and Nakayama, S.: Studies on flower initiation. I. Seitaigakukenkyu, 10, 18-27 (1947) (in Jap.). 